\title{
AVALIAÇÃO DA TENACIDADE À FRATURA DE DIFERENTES SISTEMAS CERÂMICOS
}

\author{
RELATIVE FRACTURE TOUGHNESS OF DIFFERENTS \\ DENTAL CERAMICS
}

Clovis PAGANI

Prof. Adj. do Departamento de Odontologia Restauradora da Faculdade de Odontologia de São José dos Campos - UNESP.

Carolina Baptista MIRANDA

Aluna do Curso de Pós-Graduação em Odontologia Restauradora (Nível Mestrado) da Faculdade de Odontologia de São José dos Campos - UNESP.

Marco Cícero BOTTINO

Estagiário do Departamento de Odontologia Restauradora da Faculdade de Odontologia de São José dos Campos - UNESP.

\begin{abstract}
$E$ mbora as cerâmicas possuam alta resistência à compressão, apresentam friabilidade devido à sua baixa resistência à tração e, desta forma, possuem menor capacidade de absorver impactos. Este trabalho avaliou a tenacidade à fratura de diferentes sistemas cerâmicos, que refere-se à medida da habilidade de absorção da energia de deformação de um material friável. Foram confeccionados 30 corpos-de-prova em forma de discos $(5 \mathrm{mmx} 3 \mathrm{~mm})$ utilizando-se três diferentes materiais cerâmicos, os quais foram divididos em 3 grupos: G1-10 amostras confeccionadas com a cerâmica Vitadur Alpha (Vita-Zahnfabrik); G2-10 amostras confeccionadas com a cerâmica IPS Empress 2 (Ivoclar-Vivadent) e G3-10 amostras confeccionadas com a cerâmica In-Ceram Alumina (VitaZahnfabrik). Para a obtenção dos valores de tenacidade foi utilizada a técnica da indentação que se baseia na série de fissuras que se formam sob uma carga pesada. Foram realizadas 4 impressões por amostra, utilizado um microdurômetro (Digital Microhardness Tester FM) com uma carga de 500gf, durante 10 segundos. A análise estatística dos dados (Testes ANOVA de Kruskal-Wallis e Dunn), indica que a cerâmica In-Ceram Alumina apresentou valor mediano $\left(2,96 \mathrm{~N} / \mathrm{m}^{3 / 2}\right)$, estatisticamente diferente do apresentado pela IPS Empress $2\left(1,05 \mathrm{~N} / \mathrm{m}^{3 / 2}\right)$, enquanto que a cerâmica Vitadur Alpha apresentou valores intermediários $\left(2,08 \mathrm{~N} / \mathrm{m}^{3 / 2}\right)$, sem diferenças estatísticas dos outros dois materiais. Conclui-se que as cerâmicas apresentam diferentes desempenhos de tenacidade à fratura, sendo a In-Ceram capaz de absorver maior energia comparada a Vitadur Alpha e ao IPS Empress2.
\end{abstract}

UNITERMOS: Cerâmicas odontológicas; Tenacidade à fratura; Método da indentação.

\section{INTRODUÇÃO}

O interesse e a valorização da Odontologia Estética têm sido marcantes na última década. A procura por restaurações que devolvam a função, fonética e a cor natural dos dentes tem aumentado. Assim, as cerâmicas podem ser consideradas uma excelente alternativa de material restaurador estético posterior.

Segundo Paulillo, et al. ${ }^{12}$ (1997) e Chain, et al. ${ }^{3}$ (2000), as cerâmicas constituem-se na principal alternativa de tratamento restaurador para a estrutura dental, devido à sua biocompatibilidade, resistência à compressão, condutibilidade térmica semelhante aos tecidos dentais, radiopacidade, integridade marginal, estabilidade de cor e, principalmente, elevado potencial para simular a aparência dos dentes. Além disso, este material retém menos placa bacteriana e apresenta boa resistência à abrasão (Rego, et al. ${ }^{13} 1997$; Miranda, et al. $\left.{ }^{9}, 1998\right)$. O potencial estético e a biocompatibilidade das cerâmicas podem ser considerados únicos, dentre os materiais restauradores odontológicos (Hollweg, et al. ${ }^{8}$, 1998).

Nos últimos dez anos, foram desenvolvidos novos sistemas cerâmicos que melhoraram a dureza e a estética do material, através da incorporação de vidros cerâmicos e cerâmicas com adição de cristais para reforço como o quartzo e a alumina (Hollweg, et al. ${ }^{8}$, 1998). 
Atualmente, as cerâmicas, de acordo com os procedimentos laboratoriais de fabricação, são divididas em cinco categorias: cerâmicas convencionais, fundidas, prensadas, infiltradas e computadorizadas (Rosenblum, Schulman ${ }^{14}$, 1997). As cerâmicas feldspáticas ou convencionais são constituídas basicamente de feldspato, quartzo e caulim. Apresentam-se sob forma de pó, que é misturado com água destilada ou outro veículo apropriado, sendo então esculpidas em camadas, sobre um troquel refratário, lâmina de platina ou sobre uma liga metálica (Chain, et al. ${ }^{3}$ 2000). As cerâmicas fundidas consistem em barras cerâmicas sólidas, as quais utilizam a técnica da cera perdida e centrífuga para fundição na confecção das restaurações. As cerâmicas prensadas, por sua vez, vêm na forma de blocos sólidos de cerâmica, fundidas sob alta temperatura e pressionadas dentro dos moldes criados pela técnica da cera perdida. As "cerâmicas computadorizadas" são confeccionadas a partir de blocos cerâmicos, usinados por meio de um sistema computadorizado (sistema CAD-CAM, computeraided-design - computer-aided-manufacturing). E, finalmente, as cerâmicas infiltradas são compostas por dois componentes: pó (óxido de alumínio ou corpo), o qual é fabricado como substrato poroso, e um vidro, geralmente composto por porcelana feldspática, que é infiltrado dentro do substrato poroso em alta temperatura.

De uma maneira geral, os materiais cerâmicos apresentam dois problemas relacionados com o seu uso: a formação de trincas e o desgaste no dente antagonista. A falha destes materiais ocorre devido à propagação de fendas, através do corpo da restauração. Estas fendas podem ser microscópicas e estar localizadas na superfície das restaurações (Rosenblum, Schulman ${ }^{14}$, 1997).

Embora as porcelanas possuam alta resistência à compressão, apresentam friabilidade devido à sua baixa resistência à tração. Dessa forma, possuem menor capacidade de absorver impactos e são mais susceptíveis a falhas, principalmente, antes da cimentação (Paulillo, et al. ${ }^{12}, 1997$ e Garone Netto, Burger $^{5}$, 1998).

A medida de absorção da energia de deformação de um material friável é fator crítico de intensidade do estresse ("tenacidade à fratura"), também conhecido como fator $\mathrm{k}$. A tenacidade à fratura de um material está relacionada ao nível de tensão elástica, a qual pode ser alcançada em torno da extremidade da fissura, antes do processo de fratura ser iniciado. Ou seja, é a capacidade elástica de resistir a tensões antes de gerar a fratura. Propriedades diversas como resistência mecânica, resistência ao choque térmico e susceptibilidade ao desgaste erosivo são todas fundamentalmente controladas por este parâmetro (Morena, et al. ${ }^{10}$, 1986).

A tenacidade determina todos os aspectos do comportamento mecânico de materiais friáveis. Assim, o conhecimento da tenacidade das cerâmicas dentais é um ponto inicial fundamental para a melhoria dos materiais cerâmicos utilizados em prótese dental (Morena, et al. ${ }^{10}$, 1986).

Muitas técnicas têm sido propostas com o objetivo de avaliar a tenacidade de materiais friáveis. Alguns destes métodos incluem a dupla torção, viga dupla de cantilever ("double cantilever beam"), e a técnica da indentação. A técnica da indentação já está bem estabelecida na literatura para avaliar a tenacidade de diversas cerâmicas dentais (Morena, et al. ${ }^{10}, 1986$; Rosenstiel, Porter ${ }^{15}$, 1988). Este método é considerado satisfatório no estudo dos materiais cerâmicos, uma vez que a dimensão dos espécimes é relativamente pequena e os parâmetros de desenvolvimento das fraturas são determinados por fissuras de tamanho similar às desenvolvidas na avaliação clínica (Seghi, et al. $\left.{ }^{19}, 1995\right)$. Desta forma, optamos pela utilização da técnica da indentação para a realização deste estudo.

A base da técnica de indentação para obtenção dos valores de tenacidade é a série de fissuras que se formam, sob uma carga intensa (Hassan, et al. ${ }^{7}, 1981$; Morena, et al. ${ }^{10}$, 1986; Seghi, Denry ${ }^{18}$, 1992; Seghi, et al. ${ }^{19}$, 1995). O tamanho das fissuras produzidas por tal técnica é uma função inversa da tenacidade.

O método de indentação é indicado para o estudo de materiais friáveis, apresentando como vantagem o tamanho reduzido da ponta do indentador utilizado no teste, uma vez que este permite a realização de várias medidas em uma amostra relativamente pequena (Rosenstiel, Porter ${ }^{15}$, 1988). Esta técnica tem sido utilizada para obtenção dos valores de tenacidade à fratura $\left(\mathrm{K}_{\mathrm{IC}}\right)$ de porcelanas odontológicas (Anusavice, Lee $^{1}$, 1985; Morena, et al. ${ }^{10}$, 1986; Wagner, $\mathrm{Chu}^{20}$, 1996; Scherrer, et al. ${ }^{16}$, 1998; Scherrer, et al. ${ }^{17}$, 1999; Gorman, et al. ${ }^{6}, 2000$ ).

Na prática clínica diária observam-se diferenças na resistência ao choque térmico quando diferentes cerâmicas são avaliadas, o que pode ser explicado pela distinta tenacidade desses materiais. A rápida e infreável introdução de novos sistemas restauradores cerâmicos dificulta a seleção do material mais apropriado. Sendo que, na literatura, pouco se conhece da tenacidade à fratura de diferentes sistemas cerâmicos para uso odontológico, este trabalho tem como proposta, avaliar os valores de tenacidade de 3 marcas comerciais de cerâmicas. 


\section{MATERIAL E MÉTODOS}

\section{Preparo dos espécimes}

Para a realização deste estudo foram utilizadas três marcas comerciais de sistemas cerâmicos, incluindo uma cerâmica aluminizada, Vitadur Alpha (VD/VitaZahnfabrik), uma cerâmica aluminizada infiltrada por vidro, In-Ceram Alumina (IC/Vita-Zahnfabrik), e uma cerâmica vítrea de dissilicato de lítio, IPS Empress2 (EMP2/Ivoclar-Vivadent).

A Tabela 1 apresenta as abreviações, nomes comerciais, fabricante, tamanho e percentual médio do componente cristalino de reforço.

Os corpos-de-prova foram confeccionados em forma de discos de $5 \mathrm{~mm}$ de diâmetro e $3 \mathrm{~mm}$ de altura. Os discos foram fabricados de acordo com as recomendações dos fabricantes de cada sistema cerâmico.

As amostras, 10 corpos-de-prova para cada material,portanto 30 no total,foram embutidas em resina para posterior indentação.

\section{Avaliação da tenacidade à fratura}

Para obtenção dos valores de tenacidade foi empregado um microdurômetro (Digital Microhardness Tester FM, Future-Tech) com uma carga de 500gf, durante 10 segundos. Foram realizadas quatro impressões por amostra, obtendo-se um valor de dureza e de formação de fissuras (Figura 1).

A extensão das fissuras formadas foi medida, através de um programa de computador (Image Tool for Windows - versão 2.0), levando-se em consideração o valor da sua maior extensão, obtido a partir do centro da indentação. Assim, a tenacidade foi calculada da seguinte maneira:

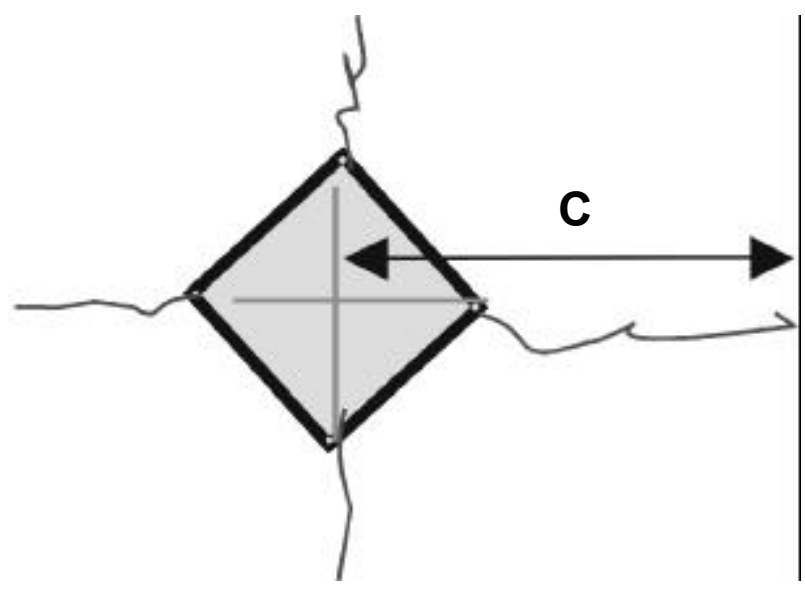

$\mathrm{C}=$ maior extensão lateral da fissura

FIGURA 1- Esquema da indentação evidenciando a formação de fissuras

$$
\mathrm{K}_{\mathrm{IC}}=\mathbf{0 , 0 1 6}(\mathrm{E} / \mathrm{H})^{1 / 2} \times \mathrm{P} / \mathrm{C}^{3 / 2} \text {, onde: }
$$

$\mathrm{K}_{\mathrm{IC}}=$ tenacidade

$\mathrm{E}=$ módulo de elasticidade $(\mathrm{GPa})$

$\mathrm{P}=$ força aplicada $(\mathrm{MN})$

$\mathrm{H}=$ dureza Vickers $(\mathrm{GPa})$

$\mathrm{C}=$ maior extensão lateral da fissura

\section{RESULTADOS}

Os dados de tenacidade (em N/m $3 / 2$ ) obtidos, segundo o material cerâmico, serão apresentados na forma de tabelas.

Na Tabela 2, mostrada a seguir, encontram-se as medidas de tendência central (média: IC-2,90; EMP21,02; VD-2,08 e mediana: IC-2,96; EMP2-1,05; VD2,08), de dispersão (desvio padrão: IC-0,43; EMP20,12 ; VD-0,38) e conjuntos de mesmo desempenho.

TABELA 1- Informações técnicas dos materiais cerâmicos

\begin{tabular}{llccc}
\hline Abreviação & $\begin{array}{c}\text { Nome } \\
\text { Comercial }\end{array}$ & $\begin{array}{c}\text { Componente cristalino } \\
\text { de reforço }\end{array}$ & Tamanho (mm) & Volume (\%) \\
\hline IC & $\begin{array}{c}\text { In-Ceram } \\
\text { Alumina }\end{array}$ & Alumina & $1-5$ & 97 \\
EMP2 & IPS Empress 2 & $\begin{array}{c}\text { Dissilicato de lítio }\left(\mathrm{SiO}_{2}-\mathrm{Li}_{2} \mathrm{O}\right) \\
\text { e ortofosfato de lítio }\left(\mathrm{Li}_{3} \mathrm{PO}_{4}\right)\end{array}$ & $0,1-5$ & 60 \\
VD & Vitadur Alpha & Alumina & n. i. & 50 \\
\hline
\end{tabular}

n.i. = não informado 
Segundo a Tabela 2, verifica-se que o menor valor mediano corresponde ao EMP2 (IPS EMPRESS 2/ Ivoclar-Vivadent), enquanto o maior valor mediano de tenacidade refere-se ao IC (In-Ceram Alumina/VitaZahnfabrik). O VD (Vitadur Alpha/Vita-Zahnfabrik) apresenta um comportamento intermediário. Observase ainda, mediante ao teste de Bartlet ( $\mathrm{p}$-valor $=0,0143$ ) que os materiais não apresentam a mesma variabilidade, ou seja, os valores de dispersão (desvio padrão) diferem entre si.

O teste estatístico não paramétrico confirma a diferença observada acima. Foi efetuada a análise de variância de Kruskal-Wallis para verificar a possibilidade de rejeitar a hipótese de igualdade dos grupos quanto à distribuição dos valores medianos. $\mathrm{O}$ resultado do teste de Kruskal-Wallis ( $\mathrm{H}=19,565$; $\mathrm{gl}=2 ; \mathrm{p}=0,001)$ indica que os grupos diferem quanto aos valores medianos. Para saber quais pares de grupos diferem entre si, foi efetuado o teste de comparação múltipla de Dunn, ao nível de significância de 5\%. Os resultados dos testes são apresentados na Tabela 3.

Verificou-se na Tabela 3 que o valor de $\mathrm{p}$ foi menor do que 0,05 , portanto, pôde-se rejeitar a hipótese de igualdade entre os três grupos em análise.

O resultado do Teste de Comparação Múltipla de Dunn, efetuado mediante o programa computacional Statistix for Windows (versão 7.0), demonstrou que a cerâmica In-Ceram apresentou maiores valores, estatisticamente diferentes dos apresentados pela Empress 2. Já a cerâmica Vitadur Alpha apresentou valores intermediários, sem diferenças estatísticas dos outros dois materiais.

\section{DISCUSSÃO}

A tenacidade à fratura é uma medida da absorção de energia de materiais friáveis, a qual está relacionada ao nível de estresse (tensão) antes da fratura ocorrer (Rosenstiel, Porter ${ }^{15}$, 1988). Segundo Fischer, Marx ${ }^{4}$ (2002) quanto maior o valor do $\mathrm{K}_{\mathrm{IC}}$, melhor é o comportamento mecânico do material. Várias características, como resistência mecânica, resistência ao choque térmico, susceptibilidade ao desgaste erosivo, são controladas por esta propriedade (Morena et al. $\left.{ }^{10}, 1986\right)$. Trata-se de um teste muito útil e simples, uma vez que, a tenacidade, está mais ligada aos fatores intrínsecos do material do que às variáveis na superfície do espécime decorrentes do preparo da amostra (Rosenstiel, Porter ${ }^{15}$, 1988).

Uma vez que as cerâmicas odontológicas são materiais praticamente não dúcteis, ou seja sem deformação plástica, e quando submetidas à tensão fraturam em dois ou mais pedaços (Campbell ${ }^{2}, 1989$ ), torna-se de grande valia a determinação da tenacidade à fratura destes materiais para melhor entendimento

TABELA 2- Estatística descritiva para os dados de tenacidade $\left(\mathrm{N} / \mathrm{m}^{3 / 2}\right)^{*}$, segundo o material cerâmico. Conjunto de mesmo desempenho (Teste de Dunn)

\begin{tabular}{lllcll}
\hline $\begin{array}{l}\text { Material } \\
\text { Cerâmico }\end{array}$ & $\mathbf{n}$ & Média & Desvio Padrão & Mediana & Conjunto** \\
\hline EMP2 & 10 & 1,02 & 0,12 & 1,05 & A \\
VD & 10 & 2,05 & 0,38 & 2,08 & A $\quad$ B \\
IC & 10 & 2,90 & 0,43 & 2,96 & B \\
\hline
\end{tabular}

* $\left(x 10^{6}\right)$

** medianas seguidas de letras iguais indicam que os conjuntos não diferem estatisticamente

TABELA 3-Resultado do teste de Kruskal-Wallis

Kruskal-Wallis

Fonte de variação

Graus de liberdade

\section{Estatística}

$\mathrm{H}$

Grupos

2

19,565

p-valor

$<0,001^{*}$

*diferença estatisticamente significante, ao nível de 0,05. 
de suas propriedades mecânicas .

Um dos métodos usados na obtenção da tenacidade à fratura $\left(\mathrm{K}_{\mathrm{IC}}\right)$ é a técnica da indentação, proposta primeiramente por Parmqvist ${ }^{11}$ em 1962 (Parmqvist, 1962 apud Rosenstiel, Porter ${ }^{15}$, 1988). A tenacidade à fratura é calculada a partir das medidas de fissuras obtidas do centro da indentação Vickers em direção às extremidades (Hassan, et al. ${ }^{7}, 1981$; Morena, et al. ${ }^{10}, 1986$; Rosenstiel, Porter ${ }^{15}$, 1988; Seghi, Denry ${ }^{18}$, 1992; Seghi, et al. $\left.{ }^{19}, 1995\right)$.

A tenacidade é dependente dos valores do módulo de elasticidade, tamanho da fissura e microdureza do substrato. Neste estudo, foram avaliadas três cerâmicas dentais (In-Ceram Alumina, IPS Empress2 e Vitadur Alpha), sendo encontrados diferentes valores. Para a determinação dos valores de tenacidade utilizamos o módulo de elasticidade de cada material cerâmico fornecido pelos fabricantes: $283 \mathrm{GPa}, 96 \mathrm{GPa}$ e $58 \mathrm{GPa}$ respectivamente para o In-Ceram Alumina (VitaZahnfabrik), IPS Empress2 (Ivoclar-Vivadent) e Vitadur Alpha (Vita-Zahnfabrik). Valores parecidos também foram empregados por Fischer \& $\mathrm{Marx}^{4}$ (2002) em que foram usados módulo de elasticidade de 96GPa para o IPS Empress2, 251GPa para a InCeram Alumina Celay e 66GPa para a Vita Omega Opaker. White et al. ${ }^{22}$ (1994), no entanto, em um estudo avaliando o módulo de ruptura de diferentes cerâmicas, utilizaram um valor do módulo de elasticidade de 57,3GPa para a cerâmica Vitadur N e 236GPa para a In-Ceram Alumina. Quando avaliando as propriedades mecânicas do IPS Empress (Ivoclar-Vivadent), Gorman, et al. ${ }^{6}$ (2000) também empregaram um módulo de elasticidade semelhante no valor de 70GPa. De acordo com Campbell ${ }^{2}$ (1989), à medida que se aumenta o módulo de elasticidade há um aumento na força necessária para ruptura, fato também por nós observado.

A partir dos nossos resultados, observamos que o material In-Ceram Alumina apresentou a maior média de tenacidade $\left(2,96 \mathrm{~N} / \mathrm{m}^{3 / 2}\right)$, enquanto a Vitadur Alpha $\left(2,08 \mathrm{~N} / \mathrm{m}^{3 / 2}\right)$ apresentou um valor intermediário e o IPS Empress2 $\left(1,05 \mathrm{~N} / \mathrm{m}^{3 / 2}\right)$ o menor valor. Embora a Vitadur Alpha apresente fissuras com tamanhos próximos ao da In-Ceram Alumina (média de 27,27 $\mu \mathrm{m}$ e $25,51 \mu \mathrm{m}$, respectivamente), a dureza e o módulo de elasticidade da In-Ceram Alumina (média de $1215,78 \mathrm{HV}$ e $283 \mathrm{GPa}$, respectivamente) são maiores do que da Vitadur Alpha (média de 569,61HV e $58 \mathrm{GPa}$, respectivamente). Conseqüentemente, a tenacidade à fratura da In-Ceram Alumina é maior. Já em relação ao IPS Empress2, este material apresentou uma média de dureza $(575,0 \mathrm{HV})$ semelhante à da Vitadur Alpha, entretanto com os maiores valores de fissuras (média de 46,8 $\mu \mathrm{m}$ ), justificado pelo seu menor módulo de elasticidade, resultando em uma menor tenacidade à fratura.

Outros autores, também estudando a tenacidade de diferentes materiais cerâmicos, através da técnica de indentação, encontraram os maiores resultados para as cerâmica aluminizadas. Wen, et al. ${ }^{21}$ (1999) apresentaram valores de tenacidade de $1,96 \mathrm{~N} / \mathrm{m}^{3 / 2}$ para o Hi-Ceram (porcelana de vidro aluminizada), 1,27N/ $\mathrm{m}^{3 / 2}$ para o IPS Empress e $4,44 \mathrm{~N} / \mathrm{m}^{3 / 2}$ para o In-Ceram Alumina. Fischer, Marx ${ }^{4}$ também avaliaram as cerâmicas IPS, In-Ceram Alumina e Vita Omega, encontrando os seguintes valores: $2,48 \mathrm{~N} / \mathrm{m}^{3 / 2}, 5,0 \mathrm{~N} /$ $\mathrm{m}^{3 / 2} \mathrm{e} 1,34 \mathrm{~N} / \mathrm{m}^{3 / 2}$, respectivamente. Valores parecidos aos por nós relatados, quando da avaliação do IPS Empress2, foram descritos por Scherrer, et al. ${ }^{16}(1998)$ e Gorman, et al. ${ }^{6}$ (2000) em trabalhos avaliando o IPS Empress. A primeira pesquisa encontrou um valor de $0,85 \mathrm{~N} / \mathrm{m}^{3 / 2}$, a segunda de $1,33 \mathrm{~N} / \mathrm{m}^{3 / 2}$, enquanto que nós encontramos um valor de $1,05 \mathrm{~N} / \mathrm{m}^{3 / 2}$. Para Fischer $\& \operatorname{Marx}^{4}$ (2002), os maiores resultados observados para a In-Ceram Alumina, ocorreram devido a sua composição microestrutural estar baseada em uma matriz de alumina infiltrada por vidro de borosilicato lantânio.

Embora, os artigos encontrados sejam concordes, mostrando os maiores valores para as cerâmicas aluminizadas, poucos artigos estão disponíveis na literatura utilizando as mesmas cerâmicas que as empregadas em nosso trabalho. Assim, são necessários mais estudos para se avaliar com mais segurança as propriedades mecânicas destes materiais.

\section{CONCLUSÃO}

A cerâmica In-Ceram apresentou maiores valores medianos de tenacidade, estatisticamente diferentes dos apresentados pela Empress 2. Já a cerâmica Vitadur Alpha apresentou valores intermediários, sem diferir estatisticamente dos outros dois materiais. Conseqüentemente, conclui-se que as cerâmicas apresentam diferentes desempenhos de tenacidade à fratura, sendo a In-Ceram capaz de absorver maior energia comparada a Vitadur Alpha e ao IPS Empress2.

\section{ABSTRACT}

Although ceramics present high compressive strength, they are brittle materials due to their low tensile strength so they have lower capacity to absorb shocks. This study evaluated the fracture toughness 
of different ceramic systems, which refers to the ability of a friable material to absorb defformation energy. Three ceramic systems were investigated. Ten cylindrical samples $(5,0 \mathrm{~mm} \times 3,0 \mathrm{~mm})$, were obtained from each ceramic material as follows: G1- 10 samples of Vitadur Alpha (Vita-Zahnfabrik); G2- 10 samples of IPS Empress2 (Ivoclar-Vivadent); G3- 10 samples of In-Ceram Alumina (Vita-Zahnfabrik). Fracture toughness values were collected upon indentation tests that were performed under a heavy load. A microhardness tester (Digital Microhardness Tester FM) utilized a $500 \mathrm{gf}$ load cell during 10seconds to perform four impressions on each sample. Statistically significant results were observed (ANOVA and Kruskal-Wallis tests). In-Ceram Alumina presented the highest median toughness values $\left(2,96 \mathrm{~N} / \mathrm{m}^{3 / 2}\right)$, followed by Vitadur Alpha $\left(2,08 \mathrm{~N} / \mathrm{m}^{3 / 2}\right)$ and IPS Empress2 $\left(1,05 \mathrm{~N} / \mathrm{m}^{3 / 2}\right)$. It may be concluded that different ceramic systems present distinct fracture toughness values, thus In-Ceram is capable of absorbing superior stress when compared to Vitadur Alpha and IPS Empress2.

UNITERMS: Dental ceramics, Fracture toughness, and Indentation method

\section{AGRADECIMENTOS}

- Ao professor Ivan Balducci pela realização da análise estatística

- Ao Laboratório Vaiazzi pela confecção dos corpos-de-prova em In-Ceram Alumina (VitaZahnfabrik) e, em Vitadur Alpha (Vita-Zahnfabrik)

- À empresa Ivoclar-Vivadent pela confecção das amostras em IPS Empress2.

\section{REFERÊNCIAS BIBLIOGRÁFICAS}

1- Anusavice KJ, Lee RB. Effect of firing temperature and water exposure on crack propagation in unglazed porcelain. J Dent Res 1985;64 [abstract 1092].

2- Campbell SD. A comparative strength study of metal ceramic and all-ceramic esthetic materials: Modulus of rupture. J Prosthet Dent 1989 Oct;62:476-9.

3- Chain MC, Arcari GM, Lopes GC. Restaurações cerâmicas estéticas e próteses livres de metal. RGO 2000 abr/jun; 48:67-70.

4- Fischer H, Marx R. Fracture toughness of dental ceramics: comparison of bending and indentation method. Dent Mat 2002;18:12-9.
5- Garone Neto N, Burger R.C. Inlay e onlay em Dentística: cimentações adesivas com cimentos resinosos. In: Todescan FF, Bottino M.A. Atualização na clínica odontológica. A prática da clínica geral. São Paulo: Artes Médicas, 1996, cap.7, p.161-90.

6- Gorman CM, McDevitt WE, Hill R.G. Comparison of two heat pressed all ceramic dental materials Dent Mat 2000;16:389-95.

7- Hassan R, Caputo AA, Bunshah R.F. Fracture toughness of human enamel. J Dent Res 1981;60: 820-27.

8- Holleg H, et al. Sistema In-Ceram: uma alternativa para a otimização estética em prótese unitária. Odonto Pope 1998; 2: 379-88.

9- Miranda CC, et al. Sistema In-Ceram Alumina. Rev Bras Prótese Clin Lab 1998; 1:163-72.

10- Morena R, Lockwood PE, Fairhurst CW. Fracture toughness of commercial dental porcelains. Dent Mater 1986; 2:58-62.

11- Parmqvist S. Occurrence of crack formation during Vickers indentation as a measure of the toughness of hard metals. Arch Eisenhuttenwes 1962;33:629-33 apud Rosenstiel SF, Porter SS. Apparent fracture toughness of dental porcelain with a metal substructure. Dent Mat 1988; 4:187-90.

12- Paulillo LAMS, Serra MC, Francischone CE. Cerâmica em dentes posteriores. ROBRAC 1997; 6:37-9.

13- Rego MA, Silva RC, Araújo M.A. Restaurações de porcelana "inlay-onlay” - caso clínico. JBC 1997 maio/jun.;1:45-49.

14- Rosemblum MA, Schulman A. A review of all-ceramic restorations. J Am Dent Assoc 1997;128:297-307.

15- Rosentiel SF, Porter SS. Apparent fracture toughness of dental porcelain with a metal substructure. Dent Mat 1988;4:187-90.

16- Scherrer SS, et al. Fracture toughness $\left(\mathrm{K}_{\mathrm{IC}}\right)$ of a dental porcelain determined by fractographic analysis. Dent Mat 1999; 15:342-8.

17- Scherrer SS, Denry IL, Wiskott H.W.A. Comparison of threefracture toughness testing techniques using a dental glass and a dental ceramic. Dent Mat 1998 July;14:246-55.

18- Seghi RR, Denry I. Effects of external bleaching on indentation and abrasion characteristics of human enamel in vitro. J Dent Res 1992; 71:1340-44.

19- Seghi RR, Denry I, Rosenstiel SF. Relative fracture toughness and hardness of new dental ceramics. J Prosthet Dent 1995 August; 74:145-50.

20- Wagner WC, Chu TM. Biaxial flexural strength and indentation fracture toughness of three new dental core ceramics. J Prosthet Dent 1996;76:140-4.

21- Wen MY, et al. Comparative mechanical property characterization of 3 all ceramic core materials. Int J Prosthod 1999;12:534-41.

22- White SN, et al. Moduli of rupture of layered dental ceramics. Dent Mat 1984 Jan;10:52-8. 
Endereço para correspondência:

Carolina Baptista Miranda

Av. Tívoli, 567 Apto 14 Vila Betânia São José dos

Campos - São Paulo

CEP:12245-230

E-mail: carolinabaptistamiranda@hotmail.com

cicbottino@hotmail.com / Marco Cícero Bottino

Fone : (12) 3921-3556 / (11) 3167-6641

Fax: (11) 3167-5634 